\title{
POLARITIES IN FINITE PROJECTIVE PLANES
}

\section{REINHOLD BAER}

Introduction. If $\pi$ is a finite projective plane, ${ }^{1}$ then every line in $\pi$ carries the same number $n+1$ of points and through every point there pass $n+1$ lines. The total number of points (lines) in $\pi$ is $n^{2}+n+1$. This integer $n(\geqq 2)$ shall retain its significance throughout this note. No further requirement is imposed upon $\pi$. In particular it is not necessary that the Theorem of Desargues is valid in $\pi$.

We are going to use the arithmetical properties of $n$, and of other suitably selected numbers, for an investigation of the polarities ${ }^{2}$ in $\pi$. In this fashion we prove the rather surprising fact that there exist always at least $n+1$ absolute points (= points lying on their polars). This lower limit is reached for even $n$ if, and only if, all the absolute points are collinear, and for odd $n$ if, and only if, no line carries more than two absolute points. If the number $M$ of absolute points is greater than $n+1$, then $n$ is a square, and every prime divisor of $n$ is a divisor of $M-1$.

A polarity will be termed regular, if any two lines which carry absolute points, but do not carry their own poles, carry the same number of absolute points. Denoting this number by $i+1$, we find $0<i^{2} \leqq n, i \equiv n$ modulo 2 and $n-1 \equiv 0$ modulo $i+1$. If $n$ is even, then $n=i^{2}$; if $n$ is odd and $\pi$ Desarguesian, then $i=1$ or $n=i^{2}$. These results are corollaries to theorems describing the distribution of elliptic and hyperbolic elements ${ }^{3}$ for a regular polarity.

As an application of these results we prove the nonexistence of hyperbolic polarities, using this term in a rather comprehensive fashion. ${ }^{4}$ This theorem constitutes a generalization of Topel's theorem ${ }^{5}$ asserting that every geometry of Bolyai-LobachevskiY is infinite.

1. The absolute elements. It is well known ${ }^{6}$ that an absolute line ${ }^{7}$

Presented to the Society, February 23, 1946; received by the editors September 2, 1945.

${ }^{1}$ See, for example, Veblen-Young [5] or Baer [1] for the elementary concepts used. Numbers in brackets refer to the Bibliography at the end of the paper.

${ }^{2}$ For a definition of "polarity" see Veblen-Young [5, p. 263].

${ }^{3}$ We follow Liebmann $[3$, pp. 36, 37] in introducing these concepts. For their definition, as used here, see $\$ 2$ below.

4 The definition of the term will be found in $\$ 3$. For a more detailed discussion of this new concept, see a forthcoming publication of the author.

5 Topel [4].

- Baer [1, lemma]. future.

${ }^{7}$ Of the polarity under consideration. We are going to omit this phrase in the 
(=line passing through its pole) carries one and only one absolute point.

LEMma. If the line $h$ is not an absolute line, then the number of points on $h$ which are not absolute is even.

Proof. The pole $H$ of $h$ is not on $h$, since $h$ is not absolute. If $P$ is a point on $h$, then denote by $P^{\prime}$ the intersection of $h$ and of the polar of $P$. The polar of $P$ is then just the line ${ }^{8} H+P^{\prime}$. Thus it follows that $P$ is absolute if, and only if, $P=P^{\prime}$, and that $P^{\prime \prime}=P$. The points on $h$ which are not absolute occur therefore in pairs, proving our contention.

THEOREM 1. If $n$ is even, then every line carries an odd number of absolute points.

PROoF. If $n$ is even, then every line carries an odd number of points. It is a consequence of the lemma that an even number of these is not absolute, and the number of absolute points is therefore odd.

Theorem 2. Assume that $n$ is odd. Then the line $h$ is absolute if, and only if, $h$ carries one and only one absolute point.

PRoof. That absolute lines carry one and only one absolute point has been remarked before. If the line $h$ is not absolute, then it follows from the lemma that the line $h$ carries an even number of absolute points, since $h$ carries an even number of points for odd $n$. This proves our contention, since 1 is not even.

Remark 1. If $n$ is even, then it may happen that the absolute points are just the $n+1$ points of a suitable line. But it follows from Theorem 1 that every line carries absolute points. Thus there will exist lines which are not absolute, though they carry one and only one absolute point.

We denote by $M$ the number of absolute points. This integer $M$ and the invariant $n$ are connected by the following fundamental congruences.

THEOREM 3. (a) $M \equiv n+1$ modulo 2 .

(b) $(M-n-1) n^{\left(p^{i}-1\right) / 2}\left(n^{(p-1) p^{i} / 2}-1\right) \equiv 0$ modulo $p^{i+1}$ for every odd prime number $p$ and every $j \geqq 0$.

Proof. If $m$ is a positive integer, then we term an $m$-cycle every ordered set ${ }^{9}$ of $m$ points $P(1), \cdots, P(m)$ with the property:

${ }^{8}$ If $P$ and $Q$ are two different points, then $P+Q$ designates the uniquely determined line passing through $P$ and $Q$.

- Note that these points need not be different. 
(*) $P(i)$ is on the polar of $P(i+1)$ for $0<i<m$ and $P(m)$ is on the polar of $P(1)$.

The $m$-cycles $P(1), \cdots, P(m)$ and $Q(1), \cdots, Q(m)$ are said to be equal if, and only if, $P(1)=Q(1), \cdots, P(m)=Q(m)$. Finally we denote by $N(m)$ the number of distinct $m$-cycles.

(i) If $2<m$, then $N(m)=\left(n^{2}+n+1\right)(n+1)^{m-2}+n N(m-2)$.

To prove this recursion formula we say that the $m-1$ points $P(1), \cdots, P(m-1)$ form an $m$-chain if $P(i)$ is on the polar of $P(i+1)$ for $0<i<m-1$. Since the point $P(1)$ of an $m$-chain may be any point in the plane, $P(1)$ may be selected in $n^{2}+n+1$ different fashions. If $P(1), \cdots, P(i)$ are already selected, then $P(i+1)$ may be any point on the polar of $P(i)$, and thus $P(i+1)$ may be chosen in $n+1$ different ways. Consequently we have shown that:

$\left(\mathrm{i}^{\prime}\right)$ the number $N^{\prime}(m)$ of different $m$-chains is $\left(n^{2}+n+1\right)(n+1)^{m-2}$.

If $P(1)=P(m-1)$, then we term the $m$-chain $P(1), \cdots, P(m-1)$ an $m$-chain of first kind; and it is an $m$-chain of second kind if $P(1) \neq P(m-1)$. If this $m$-chain is of the first kind, then $P(m-2)$ is on the polar of $P(m-1)=P(1)$, proving that the $m-2$ points $P(1), \cdots, P(m-2)$ form an $(m-2)$-cycle. Thus every $m$-chain of the first kind "begins" with an $(m-2)$-cycle, and every $(m-2)$-cycle is the beginning of one and only one $m$-chain of the first kind. Hence we have shown that:

$\left(i^{\prime \prime}\right) N(m-2)$ is the number of $m$-chains of the first kind.

If $P(1), \cdots, P(m-2), P(1)$ is an $m$-chain of the first kind, then $P(1), \cdots, P(m-2), P(1), P$ is an $m$-cycle if, and only if, $P$ is on the polar of $P(1)$. Hence there exist exactly $(n+1) N(m-2) m$-cycles which begin with an $m$-chain of the first kind.

If $P(1), \cdots, P(m-1)$ is an $m$-chain of the second kind, then $P(1) \neq P(m-1)$, and the polars of $P(1)$ and $P(m-1)$ meet in a uniquely determined point $P$. The only $m$-cycle beginning with the given $m$-chain of the second kind is, therefore, $P(1), \cdots, P(m-1)$, $P$. Thus the number of $m$-chains of the second kind equals the number of $m$-cycles which begin with an $m$-chain of the second kind. It is a consequence of $\left(i^{\prime}\right)$ and $\left(i^{\prime \prime}\right)$ that this number is

$$
\left(n^{2}+n+1\right)(n+1)^{m-2}-N(m-2) \text {. }
$$

Combining the results of the last two paragraphs we find that

$$
\begin{aligned}
N(m) & =(n+1) N(m-2)+\left(n^{2}+n+1\right)(n+1)^{m-2}-N(m-2) \\
& =\left(n^{2}+n+1\right)(n+1)^{m-2}+n N(m-2),
\end{aligned}
$$

completing the proof of (i).

(ii) $N(2 m+1)=(n+1)^{2 m+1}+n^{m}(M-n-1)$. 
If $m=0$, then we have to determine the number $N(1)$ of 1 -cycles. But the point $P(1)$ is a 1-cycle if, and only if, $P(m)=P(1)$ is on the polar of $P(1)$, and this is equivalent to saying that $P(1)$ be an absolute point. The number $N(1)$ of 1 -cycles is, therefore, just the number $M$ of absolute points. Since the right side of (ii) reduces to $M$ if $m=0$, we have verified (ii) for $m=0$.

We now prove (ii) by complete induction with regard to $m$. We infer from (i) and the induction hypothesis that

$$
\begin{aligned}
N(2 m+1)= & \left(n^{2}+n+1\right)(n+1)^{2 m-1}+n N(2 m-1) \\
= & \left(n^{2}+n+1\right)(n+1)^{2 m-1}+n(n+1)^{2 m-1} \\
& +n^{m}(M-n-1) \\
= & (n+1)^{2 m-1}\left(n^{2}+2 n+1\right)+n^{m}(M-n-1) \\
= & (n+1)^{2 m+1}+n^{m}(M-n-1),
\end{aligned}
$$

completing the proof of (ii).

(iii) $N(2 m)=(n+1)^{2 m}+(n+1) n^{m+1}$.

If $m=1$, then the right side of (iii) reduces to $(n+1)\left(n+1+n^{2}\right)$. But this is just the number of 2-cycles $P(1), P(2)$, since $P(1)$ may be selected in $n^{2}+n+1$ ways, since $P(2)$ has to be a point on the polar of $P(1)$, and since $P(1)$ is on the polar of $P(2)$ if, and only if, $P(2)$ is on the polar of $P(1)$, so that $P(2)$ may be any one of the $n+1$ points on the polar of $P(1)$.

To prove (iii) now by complete induction with regard to $m$, we deduce from (i) and the induction hypothesis that

$$
\begin{aligned}
N(2 m+2) & =\left(n^{2}+n+1\right)(n+1)^{2 m}+n N(2 m) \\
& =\left(n^{2}+n+1\right)(n+1)^{2 m}+n(n+1)^{2 m}+(n+1) n^{m+2} \\
& =(n+1)^{2 m+2}+(n+1) n^{m+2},
\end{aligned}
$$

completing the proof of (iii).

(iv) $N\left(p^{i}\right) \equiv N\left(p^{i-1}\right)$ modulo $p^{i}$ for every prime $p$ and $0<i$.

If $P(1), \cdots, P\left(p^{j-1}\right)$ is a $p^{j-1}$ cycle, then we may derive from this $p^{i-1}$-cycle a $p^{j}$-cycle by repeating it $p$ times:

$P(1), \cdots, P\left(p^{j-1}\right), \cdots, P(1), \cdots, P\left(p^{j-1}\right), \cdots, P(1), \cdots, P\left(p^{j-1}\right)$.

Consider now one of the $N\left(p^{j}\right)-N\left(p^{i-1}\right) p^{i}$-cycles which cannot be derived from a $p^{j-1}$-cycle in the indicated fashion, say the cycle $P(1), \cdots, P\left(p^{j}\right)$. Suppose that $i$ is an integer such that $0 \leqq i<p^{j}$ and such that $P(k)=P(k+i)$ for every $k$ where if necessary we reduce $k+i$ modulo $p^{i}$. If $i \neq 0$, then $i=i^{\prime} p^{h}$ where $i^{\prime}$ is prime to $p$ and $0 \leqq h<j$. The permutation: $k \rightarrow k+i$ is a cyclic permutation which 
leaves invariant the cycle $P(1), \cdots, P\left(p^{j}\right)$. Every power of this permutation leaves this cycle invariant; and thus this cycle is left invariant by the permutation: $k \rightarrow k+p^{h}$. But this is impossible, since it would imply that the cycle under consideration could be derived from a $p^{j-1}$-cycle. Hence our cycle is left invariant by the identity permutation only. But the point sets $P(i), \cdots, P\left(p^{j}\right), P(1), \cdots, P(i-1)$ are all $p^{j}$-cycles, and from what we showed just now, it follows that they are all different. Consequently we can divide the set of those $p^{j}$-cycles which cannot be derived from $p^{j-1}$-cycles into mutually exclusive sets containing $p^{i}$ cycles each. Thus the number of these cycles which is $N\left(p^{j}\right)-N\left(p^{j-1}\right)$ is divisible by $p^{j}$, proving (iv).

From (ii), (iii) and (iv) we infer that

$$
M=N(1) \equiv N(2)=(n+1)\left(n^{2}+n+1\right) \text { modulo } 2 .
$$

But $n^{2}+n+1$ is always an odd number, proving (a).

From (ii) and (iv) we infer that we have for odd primes $p$

$$
\begin{aligned}
(n+1)^{p^{j}}+n^{(p j-1) / 2}(M-n-1) & \equiv N\left(p^{j}\right) \equiv N\left(p^{j-1}\right) \\
& \equiv(n+1)^{p^{j-1}}+n^{\left(p^{j-1-1) / 2}\right.}(M-n-1) \text { modulo } p^{j} .
\end{aligned}
$$

Since $j \leqq p^{j-1}$ for $0<j$, it follows that the $p^{j-1}$ th and the $p^{j}$ th power of $n+1$ are divisible by $p^{i}$, if $n+1$ is divisible by the prime $p$. If $n+1$ is prime to $p$, then these powers of $n+1$ are congruent modulo $p^{j}$, since $p^{j}-p^{i-1}$ is the order of the multiplicative group of classes of residues modulo $p^{j}$ which are prime to $p$. Hence it follows from the above congruence that

$$
(M-n-1)\left(n^{\left(p^{i}-1\right) / 2}-n^{\left(p^{j-1}-1\right) / 2}\right) \equiv 0 \text { modulo } p^{j},
$$

and (b) is an immediate consequence of this congruence.

Remark 2. The reader might wonder why we did not state the congruences modulo $2^{j}$ which could be deduced from (iii) and (iv) with the exception of the case $j=1$, stated in (a). The reason is that these congruences are satisfied for every integer $n$-they do not involve $M$ at all-so that they do not give any new information. As a matter of fact for most of our purposes it would suffice to state the case $j=0$ of the congruences (b). But no simplification or abbreviation of our proofs could be obtained by the restriction to the case $j=0$.

The same method which we employed when proving (iv) could be used for a proof of the following generalization of (iv).

(iv') $N(m) \equiv \sum_{d} \bar{d} N(m / d)$ modulo $m$, where $1<m$ and where the summation ranges over all squarefree divisors $d$ of $m$, the number $\bar{d}$ being +1 or -1 according as the number of prime factors of $d$ is odd or even. 
But so far we have not made any applications of (iv') that could not be deduced from Theorem 3, (a) and (b).

THEOREM 4. If $p$ is a divisor of $n$, then $M \equiv 1$ modulo $p$.

Proof. For $p=2$ we deduce this from Theorem 3, (a). If $p$ is odd, then considering the special case $j=0$ of Theorem 3 , (b) we obtain $0 \equiv(M-n-1)\left(n^{(p-1) / 2}-1\right) \equiv 1-M$ modulo $p$, proving our contention.

Remark 3. From $1<n$ it follows that $n$ is divisible by certain primes; and Theorem 4 shows, therefore, the existence of absolute points. ${ }^{10}$ We improve upon this statement in the next theorem.

THEOREM 5. There exist at least $n+1$ absolute points.

Proof. If $n$ is even, then we deduce from Theorem 1 the existence of absolute points on every line. Consider a point $P$ which is not absolute (every absolute line carries $n$ of them). Then each of the $n+1$ lines through $P$ carries an absolute point, but no two of these lines through $P$ have an absolute point in common. Thus there exist at least $n+1$ absolute points.

If $n$ is odd, then there exists by Theorem 4 -as has been pointed out in Remark 3-an absolute point $H$. Denote by $h$ the polar of $H$. Then $h$ passes through $H$. If $k$ is a line through $H, k \neq h$, then it follows from Theorem 2 that $k$ carries an absolute point, different from $H$. Since there pass through $H$ exactly $n$ lines apart from $h$, we have shown the existence of the absolute point $H$ and of $n$ further absolute points.

Corollary 1. No line carries more than two absolute points if, and only if, $n$ is odd and the number $M$ of absolute points is $n+1$.

Proof. Suppose first that no line carries more than two absolute points. Since there exist by Theorem 5 at least $n+1$ absolute points, there exist lines carrying exactly two absolute points. It follows from the lemma that $n$ is odd. If $H$ is some fixed absolute point, then we infer from Theorem 2 that $H$ is the one and only absolute point on the polar $h$ of $H$, and that every line $k \neq h$ passing through $H$ carries one and only one absolute point different from $H$. Since every absolute point may be connected with $H$ by a line, this implies that the number of absolute points is $n+1$.

Assume conversely that $n$ is odd and $M=n+1$. If the line $h$ carries an absolute point $P$, then either $h$ is an absolute line and $P$ its pole

${ }^{10}$ The corresponding theorem for projectivities is trivial, since every involutorial projectivity possesses fixed points. 
or else the polar $w$ of $P$ is an absolute line, passing through $P$, and $w \neq h$. But each of the lines different from $w$ and passing through $P$ carries by Theorem 2 at least one further absolute point. Since there pass through $P$ just $n$ lines different from $w$, since different lines through $P$ meet in $P$, and since there exist exactly $n$ absolute points different from $P$, it follows that the lines through $P$ which are different from $w$ carry just one absolute point different from $P$. This result applied to $h$ proves that $h$ carries at most two absolute points.

COROLlaRY 2. The following properties imply each other:

(i) $n$ is even and $M=n+1$.

(ii) There exists a line all of whose points are absolute.

(iii) All the absolute points are collinear.

Proof. Suppose that (i) is satisfied and that the line $h$ carries a point $P$ which is not absolute. It is a consequence of Theorem 1 that every line carries absolute points. The $n+1$ lines through $P$ have no absolute point in common and carry each at least one absolute point which is necessarily different from $P$. Hence every line through $P$ carries one and only one absolute point, since only $n+1$ absolute points are available. Thus we have shown that a line carries one and only one absolute point if, and only if, not all of its points are absolute. Since there exist, by Theorem 5 , at least two different absolute points, and since these may be connected by a line, this line carries absolute points only, and we have shown that (ii) is a consequence of (i).

If $h$ is a line all of whose points are absolute, then it is impossible that a point outside $h$ is absolute, ${ }^{11}$ proving that (iii) is a consequence of (ii).

Assume finally the collinearity of the absolute points. Then there exists a line $h$ which carries all the absolute points. Since there are $n+1$ points on $h$, and since by Theorem 5 there exists at least $n+1$ absolute points, it follows that every point on $h$ is absolute and that $M=n+1$. If $k$ is a line different from $h$ which does not pass through the pole of $h$, then $k$ is not absolute, though its one and only absolute point is its intersection with $h$. Hence $n$ is even by the lemma, proving that (i) is a consequence of (iii).

Remark 4. Conditions (ii) and (iii) may be combined into the following condition which is equivalent to them.

(iv) There exists one and only one line all of whose points are absolute.

"See Baer [1, lemma]. 
Remark 5. Corollaries 2 and 1 show the impossibility of improving Theorem 5, since it is easy to construct polarities in finite Desarguesian planes which meet the requirements of these corollaries; see the Appendix to $\$ 2$.

THEOREM 6. If there exist more than $n+1$ absolute points, then $n$ is a square.

Proof. If $n$ is not a square. then there exists ${ }^{12}$ an infinity of odd prime numbers $p$ such that $n$ is not a quadratic residue modulo $p$. If $p$ is such a prime number, then $n$ is prime to $p$ and ${ }^{13} n^{(p-1) / 2} \not \equiv 1$ modulo $p$. Since $n^{(p-1) / 2}-1$ is prime to $p$, it follows from Theorem 3 , (b) that $M \equiv n+1$ modulo $p$ for each odd prime number $p$ such that $n$ is not a quadratic residue modulo $p$. Since there exists an infinity of such primes, we have $M=n+1$, if $n$ is not a square, as we claimed.

The following alternative proof may be of interest. There exists, if $n$ is not a square, at least one odd prime $p$ such that $n$ is not a quadratic residue modulo $p$. Then $n$ and $n^{(p-1) p^{i} / 2}-1$ for $0 \leqq j$ are prime to $p$. Hence it follows from Theorem 3 , (b) that $M-n-1 \equiv 0$ modulo $p^{i}$ for every positive $j$, proving that $M=n+1$.

THEOREM 7. There exist at least $n(n+1) / 2$ points which are not absolute, but are on absolute lines. ${ }^{14}$

Proof. There exist by Theorem 4 at least $n+1$ absolute lines. If $h(1), \cdots, h(n+1)$ are $n+1$ distinct absolute lines, then each of them carries $n$ points which are not absolute. The line $h(j)$ meets each of the lines $h(1), \cdots, h(j-1)$ in a point which is not absolute. Thus there are on $h(j)$ at least $n-j+1$ points which are not absolute and which are not on the lines $h(1), \cdots, h(j-1)$. This assures us of the existence of at least $n+(n-1)+\cdots+1=n(n+1) / 2$ distinct points on the absolute lines $h(1), \cdots, h(n+1)$ which are not absolute, proving our contention.

Remark 6. It is a consequence of Theorem 1 of the next section that the lower limit given in the present theorem may actually be reached.

Corollary 3. There exist at least $(n+1)(n+2) / 2$ points which are on absolute lines.

This is an immediate consequence of Theorems 4 and 7 .

It is of interest to note that the limit given in Theorem 7 accounts

${ }^{12}$ See, for example, Hecke [2, p. 199, Satz 147].

${ }^{13}$ See, for example, Hecke [2, p. 57, (31)].

${ }^{14}$ Such points will be termed hyperbolic points. 
for just less than half of the points in the plane, and that more than half of the points of the plane are accounted for by Corollary 3 .

2. Regular polarities. It will be convenient to introduce the following notation: $:^{15}$ If the line $h$ is not absolute, but carries absolute points, then $h$ is an elliptic line; dually the point $H$ is termed a hyperbolic point, if $H$ is not absolute, though absolute lines pass through $H$. Clearly the pole of an elliptic line is hyperbolic and the polar of a hyperbolic point is elliptic.

It is a consequence of Theorem $5, \S 1$, that there always exist two different absolute points. The line connecting them is certainly not absolute, since it carries at least two absolute points; and thus there exist always elliptic lines and hyperbolic points. The lines carrying more than one absolute point are all elliptic lines. The converse is true, if $n$ is odd (Theorem 2, $\$ 1$ ); but if $n$ is even, then there may exist elliptic lines carrying one (or $n+1$ ) absolute points (Corollary 2 , $\S 1)$.

The polarity under consideration shall be termed regular if any two elliptic lines carry the same number of absolute points. The existence of irregular polarities may be inferred from Corollary $2, \S 1$. On the other side it is easy to show that the polarity is regular whenever $n$ is odd and the Theorem of Desargues (or an even weaker hypothesis) is valid. Whether or not it suffices to assume that $n$ be odd or that the absolute points are not collinear, in order to assure regularity, is still an open question.

As has been pointed out before, there exist always two different absolute points. Assuming now, as we shall do throughout this section, the regularity of our polarity, it follows that the number of absolute points on an elliptic line cannot be less than 2. Thus there exists a positive number $i$ such that every elliptic line carries exactly $i+1$ absolute points; and it is an immediate consequence of the lemma of $\$ 1$ that $i \equiv n$ modulo 2 .

THEOREM 1. The number of absolute points is in +1 , the number of hyperbolic points is $(i n+1) n(i+1)^{-1}$, and the number of points that are neither absolute nor hyperbolic is $n\left(n-i^{2}\right)(i+1)^{-1}$.

Proof. The existence of absolute points is a consequence of Theorem 3 , §1. If $H$ is an absolute point, $h$ its polar and $k \neq h$ a line through $H$, then $k$ is not absolute, since absolute lines carry one and only one absolute point. Consequently $k$ is elliptic and carries, therefore, exactly $i$ absolute points which are different from $H$. Since there pass

\footnotetext{
${ }^{15}$ Liebmann [3, pp. 36, 37].
} 
exactly $n$ lines through $H$ which are different from $h$, since different lines through $H$ have only $H$ in common, and since every absolute point, not $H$, is on a line through $H$ which is different from $h$, it follows that in +1 is the number of absolute points.

If $h$ is some absolute line, then its pole $H$ is the only absolute point on $h$, and all the other $n$ points on $h$ are hyperbolic. Since every hyperbolic point lies on exactly $i+1$ absolute lines, and since the number of absolute lines has been shown to be in +1 , it follows that $(i n+1) n(i+1)^{-1}$ is the number of hyperbolic points.

There exist altogether $n^{2}+n+1$ points of which $i n+1$ are absolute and $(i n+1) n(i+1)^{-1}$ are hyperbolic. The number of points that are neither absolute nor hyperbolic is therefore

$$
\begin{aligned}
& n^{2}+n+1-(i n+1)-(i n+1) n(i+1)^{-1} \\
&= n^{2}+n-i n-(i n+1) n(i+1)^{-1} \\
&= {[(n+1-i)(i+1)-(i n+1)] n(i+1)^{-1} } \\
&=\left(n-i^{2}\right) n(i+1)^{-1} .
\end{aligned}
$$

THEOREM 2. Every elliptic line carries $(n-1) i(i+1)^{-1}$ hyperbolic points and $\left(n-i^{2}\right)(i+1)^{-1}$ points that are neither absolute nor hyperbolic.

Proof. Denote by $j$ the number of hyperbolic points on the elliptic line $h$. Each of these lies on exactly $i+1$ absolute lines. Of all the $i n+1$ absolute lines (Theorem 1) exactly $i+1$ meet the elliptic line $h$ in absolute points; and thus we have

$$
\text { in }+1-(i+1)=j(i+1) \text { or } j=(n-1) i(i+1)^{-1} \text {, }
$$

as we claimed.

Since every elliptic line carries altogether $n+1$ points of which $i+1$ are absolute and $(n-1) i(i+1)^{-1}$ are hyperbolic, it follows that the number of points on an elliptic line that are neither absolute nor hyperbolic is exactly

$$
\begin{aligned}
n+1- & (i+1)-(n-1) i(i+1)^{-1} \\
= & {[(n-i)(i+1)-(n-1) i](i+1)^{-1}=\left(n-i^{2}\right)(i+1)^{-1} . }
\end{aligned}
$$

Corollary 1. (a) $i+1$ is a divisor of $n-1$.

(b) $i^{2} \leqq n$.

(c) $i^{2} \neq n$ implies $i^{2}+i+1 \leqq n$.

(d) $i^{2}=n$ if, and only if, every point is either absolute or hyperbolic.

(e) $i^{2}=n$ if $n$ is even.

(f) $n$ is a square if $i \neq 1$. 
Proof. It is a consequence of the first statement of Theorem 2 that $i+1$ is a divisor of $(n-1) i$. But $i$ and $i+1$ are relatively prime, showing that $i+1$ is a divisor of $n-1$.

It is a consequence of the second statement of Theorem 2 that $\left(n-i^{2}\right)(i+1)^{-1}$ is a not negative integer. Hence $0 \leqq n-\imath^{2}$, proving (b); and $n \neq i^{2}$ implies $1 \leqq\left(n-i^{2}\right)(i+1)^{-1}$ or $i+1 \leqq n-i^{2}$, proving (c).

It is a consequence of the last statement of Theorem 1 that $i^{2}=n$ is equivalent to saying that the number of points that are neither absolute nor hyperbolic is 0 , proving (d).

If $n$ is even, then we infer from Theorem $1, \S 1$, that every point is on an absolute line so that every point is either absolute or hyperbolic. Hence (e) is a consequence of (d). ( $f$ ) is an immediate consequence of Theorem 1 and Theorem $6, \S 1$.

THEOREM 3. On a line that is neither absolute nor elliptic there are $(i n+1)(i+1)^{-1}$ hyperbolic points and $(n+i)(i+1)^{-1}$ points that are neither absolute nor hyperbolic.

Proof. If the line $h$ is neither absolute nor elliptic, then none of the points on $h$ is absolute. Denote by $m$ the number of hyperbolic points on $h$. Since each hyperbolic point is on exactly $i+1$ absolute lines, and since the number of absolute lines is in +1 (Theorem 1), it follows that $i n+1=m(i+1)$, proving our first contention.

The number of points on $h$ that are neither absolute nor hyperbolic is therefore

$$
\begin{aligned}
n+1-(i n+1)(i+1)^{-1} & =[(n+1)(i+1)-(i n+1)](i+1)^{-1} \\
& =(n+i)(i+1)^{-1} .
\end{aligned}
$$

It is customary to term lines $h$ and $k$ perpendicular if $h$ passes through the pole of $k$ and $k$ passes, therefore, through the pole of $h$. A point may be termed ${ }^{16}$ elliptic if it is the intersection of two perpendicular elliptic lines. Dually the polars of elliptic points are termed hyperbolic lines.

TheOREM 4. Every elliptic line carries at least $(n-1) i(i+1)^{-1}$ elliptic points.

PRoof. If the line $h$ is elliptic, then the pole $H$ of $h$ is hyperbolic. Dualizing Theorem 2 we find that there pass exactly $(n-1) i(i+1)^{-1}$ elliptic lines through $H$. All these are perpendicular to $h$, and they meet $h$ in $(n-1) i(i+1)^{-1}$ different points which are clearly elliptic, proving our contention.

${ }^{16}$ This is a change from the terminology used by Liebmann [3, pp. 36, 37]. 
COROLLARY 2. There are at least $(i-1)(i+n)(i+1)^{-1}$ points on every elliptic line which are at the same time elliptic and hyperbolic.

Proof. We make first the obvious remark that absolute points are never elliptic. If $h$ is an elliptic line, then it carries $n+1-(i+1)$ $=n-i$ points that are not absolute, and of these $(n-1) i(i+1)^{-1}$ are hyperbolic (Theorem 2 ) and at least $(n-1) i(i+1)^{-1}$ are elliptic (Theorem 4 ). Thus the number of points on $h$ that are at the same time elliptic and hyperbolic is not less than

$$
\begin{aligned}
-(n-i) & +2(n-1) i(i+1)^{-1} \\
& =-[(n-i)(i+1)-2(n-1) i](i+1)^{-1} \\
& =\left(i^{2}-i+n i-n\right)(i+1)^{-1}=(i-1)(i+n)(i+1)^{-1} .
\end{aligned}
$$

Note that the lower limit given in Corollary 2 is 0 if, and only if, $i=1$.

THEOREM 5. If $1<i$, then every point is hyperbolic or absolute or elliptic. ${ }^{17}$

PRoof. If the point $P$ is neither absolute nor hyperbolic, then there pass, by Theorem 3, exactly $(i n+1)(i+1)^{-1}$ elliptic lines through $P$. But

$$
\begin{aligned}
(i n+1) & (i+1)^{-1}-(n+1) / 2 \\
= & (i+1)^{-1}[2(i n+1)-(i+1)(n+1)] / 2 \\
= & (i+1)^{-1}(i n+1-i-n) / 2=(i+1)^{-1}(n-1)(i-1) / 2
\end{aligned}
$$

is certainly a positive number, since $1<i$. Hence $(n+1) / 2$ $<(i n+1)(i+1)^{-1}$, proving that more than half of the lines through $P$ are elliptic. Since $P$ is not situated on any absolute line, there exist exactly $(n+1) / 2$ pairs of perpendicular lines, meeting in $P$. Since more than half of the lines through $P$ are elliptic, one of these pairs of perpendicular lines must consist of elliptic lines. Hence $P$ is elliptic, proving our theorem.

Remark. If $n$ is even, then it follows from Theorem $1, \S 1$, that every point is on an absolute line. Thus for even $n$ every point is absolute or hyperbolic. Likewise every line is absolute or elliptic, proving that for even $n$ hyperbolic and elliptic points are the same.

Theorem 6. The following three conditions are necessary and sufficient for the absence of points that are at the same time hyperbolic and elliptic.

(a) $i=1$.

\footnotetext{
${ }^{17}$ The word "or" used here should not be understood in the exclusive sense.
} 
(b) If $P$ is an elliptic point on the elliptic line $h$, then the line through $P$ perpendicular to $h$ is elliptic.

(c) Every point is elliptic or absolute or hyperbolic.

Proof. Assume first the absence of points that are at the same time hyperbolic and elliptic. Then we infer $i=1$ from Corollary 2 . If $h$ is an elliptic line, then its pole $H$ is a hyperbolic point. From $i=1$ and Theorem 2 we infer now that the number of absolute points on $h$ is 2 , and that there are exactly $(n-1) / 2$ hyperbolic points on $h$. We deduce from Theorem 4 the existence of not less than $(n-1) / 2$ elliptic points on $h$. Since the classes of elliptic, absolute and hyperbolic points are mutually exclusive, and since there are $n+1=2+(n-1) / 2$ $+(n-1) / 2$ points on $h$, we have shown that $(n-1) / 2$ is the number of elliptic points on $h$. It follows from Theorem 2 that there pass through $H$ exactly $(n-1) / 2$ elliptic lines. Each of them is perpendicular to $h$, and they meet $h$ in $(n-1) / 2$ different elliptic points. Thus a point $P$ on $h$ is elliptic if, and only if, ${ }^{18} P+H$ is elliptic, showing the necessity of (b). If finally the point $Q$ is neither absolute nor hyperbolic, then no absolute line passes through $Q$. There exist absolute points. Thus, connecting $Q$ to an absolute point, we obtain an elliptic line through $Q$. But we have shown that the $(n-1) / 2$ points on an elliptic line, that are neither absolute nor hyperbolic, are elliptic, proving that $Q$ is elliptic. This completes the proof of the necessity of the three conditions (a) to (c).

We assume conversely the validity of the conditions (a) to (c). If the point $P$ is elliptic, then there exists, by definition, a pair of perpendicular elliptic lines $h, k$ which meet in $P$. The pole $H$ of $h$ is a hyperbolic point, and we infer from (b) that a point $Q$ on $h$ is elliptic if, and only if, $H+Q$ is an elliptic line. We infer from (a) that there are on $h$ exactly two absolute points, and it follows from Theorem 2 that the number of hyperbolic points on $h$ is $(n-1) / 2$, and that the number of elliptic lines, through $H$ is $(n-1) / 2$. Since the elliptic points on $h$ are just the intersections of $h$ with elliptic lines through $H$, it follows that $(n-1) / 2$ is the number of elliptic points on $h$. Since there are on $h$ just $n+1=2+(n-1) / 2+(n-1) / 2$ points, we infer from (c) the impossibility of the existence of an elliptic point on $h$ which is at the same time hyperbolic, completing the proof.

Remark. That (c) is not a consequence of (a) and (b), and that (a) is not a consequence of (b) and (c), will be shown in the Appendix. Whether or not (b) is a consequence of (a) and (c) is still an open question.

\footnotetext{
18 See footnote 8.
} 
COROLlARY 3. If no point is at the same time hyperbolic and elliptic, and if the two perpendicular lines $h$ and $k$ meet in the point $P$, then (i) $P$ is elliptic, provided both lines $h$ and $k$ are elliptic or $h$ and $k$ are both hyperbolic; and (ii) $P$ is hyperbolic, provided one of the lines $h$ and $k$ is elliptic and the other one is hyperbolic.

Proof. That $P$ is elliptic if $h$ and $k$ are elliptic is the definition of elliptic points, and that $P$ is hyperbolic if one of the lines $h$ and $k$ is elliptic, though the other one is hyperbolic, may be inferred from Theorem 6, (b). Assume finally that $P$ is a hyperbolic point. Then it follows from Theorem 6 , (a) that exactly two absolute lines pass through $P$; and it follows from Theorem 2 that $(n-1) / 2$ is the number of elliptic lines passing through $P$. Since hyperbolic lines cannot be absolute or elliptic, the number of hyperbolic lines through $P$ is $(n-1) / 2$ too. Every elliptic line through $P$ is perpendicular to a hyperbolic line through $P$, since $P$ is not elliptic, and since lines that are neither absolute nor elliptic are hyperbolic by Theorem 6, (c). Thus every hyperbolic line through $P$ is perpendicular to an elliptic line through $P$, since elliptic and hyperbolic lines through $P$ are equal in number. The condition, stated under (ii), is therefore necessary and sufficient for $P$ to be hyperbolic, and (i) is an immediate consequence of this fact.

COROLlary 4.4 is a divisor of $n+1$ if no point is at the same time elliptic and hyperbolic.

Proof. If $P$ is an elliptic point, then we infer from Theorem 6, (a), and Theorem 2 that $(n+1) / 2$ elliptic lines pass through $P$, and we deduce from Corollary 3 , (i), that the elliptic lines passing through $P$ occur in pairs of perpendicular lines. Thus $(n+1) / 2$ is even and $n+1$ is divisible by 4 .

Appendix. The Desarguesian case. To illustrate the preceding considerations we collect here without proofs ${ }^{19}$ the main facts for the special case that the Theorem of Desargues holds in the finite projective plane under consideration. Then it is well known that the projective plane may be represented by means of coordinates from a finite field $F$. This field $F$ is commutative and contains $p^{m}$ elements for $p$ a suitable prime. Every line carries $p^{m}+1$ points so that $n=p^{m}$.

A. $p=2$.

Then there arise two possibilities. Either a!l the absolute points are collinear or else the polarity is regular with $i^{2}=n$.

${ }^{19}$ Most of the proofs are simple and immediate. Some of them will be given in a forthcoming study of polarities in Desarguesian projective planes. 
B. $p$ is odd.

Then the polarity is always regular, and the point $P$ on the elliptic line $h$ is elliptic if, and only if, the line through $P$ perpendicular to $h$ is elliptic. The number $i$ is either 1 or $i^{2}=n$. If $i=1$, then points are at the same time elliptic and hyperbolic if, and only if, -1 is a square in $F$, and this is the case if, and only if, $p^{m}-1$ is divisible by 4 . Thus $n+1$ is divisible by 4 if, and only if, -1 is not a square in $F$, and we have shown, by Theorem $6, \S 2$ :

No point is at the same time elliptic and hyperbolic if, and only if, $i=1$ and 4 is a divisor of $n+1$.

3. Hyperbolic polarities. The polarity under consideration shall be termed hyperbolic, ${ }^{20}$ if there exists a nonvacuous set of points, called the interior points, subject to the following requirements:

(i) The point $P$ is an interior point if, and only if, the points on its polar are neither interior nor absolute points.

(ii) The point $P$ is neither interior nor absolute if, and only if, there exist interior points on its polar.

(iii) ${ }^{21}$ If the line $h$ passes through the interior point $P$, then there exists an interior point $Q \neq P$ on $h$.

(iv) If $h$ and $k$ are perpendicular lines, and if each of these lines carries interior points, then $h$ and $k$ meet in an interior point.

It is known ${ }^{22}$ that, in general, (iv) is not a consequence of the first three postulates. Whether or not (iv) may be deduced from (i) to (iii) in case the projective plane is finite is still an open question.

It will be convenient to term a line interior if it carries interior points, and to term elements exterior if they are neither absolute nor interior. It is readily seen that the polar of an exterior point is an interior line, that the pole of an exterior line is an interior point, that elliptic points and lines are interior, and that hyperbolic points and lines are exterior.

The main result of this section is the following theorem.

THEOREM. ${ }^{23}$ There does not exist a hyperbolic polarity in a finite plane.

The proof will be effected in several steps.

I. Any two interior lines carry the same number of interior points.

Proof. Assume first that the interior lines $h$ and $k$ are not per-

${ }^{20}$ For a justification and discussion of this term, see a forthcoming publication of the author.

${ }^{21}$ No use will be made of this condition in the proof of the theorem below.

${ }_{22} \mathrm{~A}$ proof of this fact will be given in a forthcoming publication.

${ }^{23}$ This is an extension of Topel's theorem that the planes of Bolyai-Lobachevskir are infinite; see Topel [4]. 
pendicular. We may assume without loss in generality that the number $m$ of interior points on $h$ is not less than the number $m^{\prime}$ of interior points on $k$. Denote by $K$ the pole of $k$. If $J$ is an interior point on $h$, then $J \neq K$, since $K$ is exterior, $J+K$ is an interior line perpendicular to $k$; and it follows from (iv) that $J+K$ and $k$ meet in an interior point $J^{\prime}$. If $J$ and $Q$ are different interior points on $h$, then $K$ is not on $J+Q=h$ so that the interior lines $K+J$ and $K+Q$, perpendicular to $k$, meet $k$ in different points $J^{\prime}$ and $Q^{\prime}$ respectively. Thus there exist at least $m$ interior points on $k$, proving $m^{\prime} \leqq m \leqq m^{\prime}$ or $m=m^{\prime}$. This proves our contention for interior lines that are not perpendicular.

Assume now that $h$ and $k$ are perpendicular interior lines. They meet by (iv) in an interior point $P$. Every line through $P$ is interior, and there exist $n+1$ lines through $P$. Hence there exists an interior line $q$ through $P$ which is different from $h$ as well as $k$. Since $h, k, q$ are copunctual, and since $h$ and $k$ are perpendicular, $q$ is not perpendicular to either $h$ or $k$. Hence it follows from what we showed in the first paragraph of this proof that the number of interior points on $h$ is the same as the number of interior points on $q$ is the same as the number of interior points on $k$, and this completes the proof of $\mathrm{I}$.

We infer from I the existence of an integer $m(\geqq 0)$ such that:

$I^{\prime}$. Every interior line carries exactly $m+1$ interior points.

Next we prove:

II. Every interior line carries exactly $m+1$ exterior points.

Proof. If $P$ is an interior point on the interior line $h$, then the polar of $P$ carries only exterior points by (i). Thus the polar of $P$ meets $h$ in the exterior point $P^{\prime}$. If $H$ is the pole of $h$, then $H$ is not on $h$, and $H+P^{\prime}$ is the polar of $P$. Since the polars of points on $h$ have only the pole $H$ of $h$ in common, one verifies now that $h$ carries at least $m+1$ exterior points.

If $Q$ is an exterior point on $h$, then its polar is an interior line (by definition of exterior point) which passes through the pole $H$ of $h$. Thus the polar of $Q$ is perpendicular to $h$ and interior. It follows from (iv) that the polar of $Q$ meets $h$ in an interior point $Q^{\prime}$. One verifies as before that the number $m+1$ of interior points on $h$ cannot be less than the number of exterior points on $h$. Our contention II is an immediate consequence of the results of the two paragraphs of this proof.

III. Every interior line carries $n-2 m-1$ absolute points.

PROOF. Every line carries $n+1$ points. Every point on an interior line is either interior or absolute or exterior, and these three classes 
are mutually exclusive. Now III is an immediate consequence of $\mathrm{I}^{\prime}$ and II.

III'. The polarity is regular with $i=n-2 m-2$.

This is an almost immediate consequence of III, since exterior lines do not carry absolute points.

It has been pointed out before that elliptic points are interior and hyperbolic points are exterior. We infer from (i) that interior points cannot be exterior. Hence elliptic points cannot be hyperbolic. Thus it follows from III' and Theorem 6, (a), $\$ 2$, that $n-2 m-2=i=1$, and it follows from III that every interior line carries exactly two absolute points. Since all the $n+1$ lines passing through some interior point are interior, and since these have only an interior point in common, this implies the existence of $2(n+1)$ absolute points. On the other hand it follows from Theorem $1, \S 2$, that there exist exactly $i n+1=n+1<2(n+1)$ absolute points, a contradiction proving our theorem.

\section{BIBLIOGRAPHY}

1. R. Baer, Null systems in projective space, Bull. Amer. Math. Soc. vol. 51 (1945) pp. 907-910.

2. E. Hecke, Theorie der algebraischen Zahlen, Leipzig, 1923.

3. H. Liebmann, Synthetische Geometrie, Leipzig and Berlin, 1934.

4. B. J. Topel, Bolyai-Lobachevsky planes with finite lines, Reports of a Mathematical Colloquium, 2d series, vols. 5, 6, pp. 40-42.

5. O. Veblen and J. W. Young, Projective geometry, vol. 1.

UNIVERSITY OF ILLINOIS 